\title{
Vandetanib (ZD6474) induces antiangiogenesis through mTOR-HIF-I alpha-VEGF signaling axis in breast cancer cells
}

This article was published in the following Dove Press journal: OncoTargets and Therapy

\author{
Ling $\mathrm{Li}^{1, *}$ \\ Jingkui $\mathrm{Yu}^{2, *}$ \\ Shuhong Jiao' \\ Wei Wang' \\ Fen Zhang' \\ Shiqing Sun' \\ 'Department of Oncology, Affiliated \\ Tengzhou Central People's Hospital of \\ Jining Medical University, Zaozhuang, \\ Shandong, China; ${ }^{2}$ Breast Surgery \\ Department, Affiliated Tengzhou \\ Central People's Hospital of Jining \\ Medical University, Zaozhuang, \\ Shandong, China \\ *These authors contributed equally \\ to this work
}

Objective: Vandetanib, also known as ZD6474, has recently been proved to be a clinical drug for cancer by targeting vascular endothelial growth factor receptor 2 (VEGFR2), EGFR, and RET tyrosine kinases. We hypothesized that vandetanib will be a drug candidate for breast cancer treatment by targeting angiogenesis.

Materials and methods: Vandetanib was used to treat different breast cancer cell lines, and its effect on growth, apoptosis, and cell cycle was studied by MTT assay and flow cytometry. VEGF level in culture medium was measured by ELISA. Gene expression of mechanistic target of rapamycin (mTOR), hypoxia-inducible factor (HIF)-1 alpha, and VEGF at mRNA and protein level were analyzed by quantitative real-time-PCR and Western blot. The cellular behavior variations were investigated by using wound healing assay, transwell invasion assay, and tubular formation assay as well as experiments in vivo.

Result: We found that vandetanib can inhibit breast cancer cell line growth via apoptosis and cell cycle regulation. VEGF secretion decreases upon treatment. Vandetanib can reduce both mRNA and protein level of mTOR, HIF-1 alpha, and VEGF. Angiogenesis assays showed that vandetanib can inhibit wound healing, invasion, and tubular formation in culture. Furthermore, vandetanib inhibited the growth of breast tumor in vivo.

Conclusion: In short, our study showed that vandetanib can control angiogenesis of breast cancer in culture via mTOR, HIF-1 alpha, and VEGF signaling pathway.

Keywords: vandetanib, HIF-1 alpha, mTOR, VEGF, breast cancer

\section{Introduction}

Breast cancer is the most common malignancy in women and it is still a big challenge for treatment and management. ${ }^{1,2}$ The heterogeneity of cancer cells enable the certain cancer cells to survive treatment, proliferate, and come back again. ${ }^{1-3}$ These new cancer cells can survive in all available treatments and progress rapidly. ${ }^{1-3}$ Chemical therapy and radiotherapy all target proliferating cells while the survival malignant cells are resistant to similar treatments. ${ }^{3}$ It seems that there is no way to treat such malignancy without new methods. ${ }^{4}$ Some target therapies work well at the beginning but face the same issue. ${ }^{5}$ Therefore, we should have more alternative approaches to fight against new malignant cells. ${ }^{5}$

Among all the mechanisms to control cancer cells, one of them is to control tumor growth via energy availability supplied by neo-angiogenesis. ${ }^{6,7}$ If neo-angiogenesis is under control, the tumor size and progression of malignancy will be under control as well. ${ }^{7,8}$ Therefore, neo-angiogenesis is a target for breast cancer for improve current treatment regime. ${ }^{6}$
Correspondence: Ling Li Department of Oncology, Affiliated Tengzhou Central People's Hospital of Jining Medical University, No I8I Xingtan Road, Tengzhou, Zaozhuang 277599 , Shandong, China

Tel +86 I38 63260929

Email lilinglingling9@।63.com 
Recent studies showed that mechanistic target of rapamycin (mTOR), hypoxia-inducible factor (HIF)-1 alpha, and vascular endothelial growth factor (VEGF) are upregulated in breast cancer. ${ }^{9}$ These genes are crucial for angiogenesis under hypoxia condition. Hypoxia usually causes cells death for normal tissue but not for cancer cells. Under hypoxia, the cancer cells with ability to upregulate angiogenesis genes can survive, proliferate, and grow. ${ }^{10,11}$ On the one hand, the PI3K/AKT upregulated upon hypoxia will enhance the expression of mTOR, which will promote the HIF-1 alpha signaling pathway eventually. ${ }^{12,13}$ On the other hand, mitogen-activated protein kinase (MAPK), another target of hypoxia, will increase p38 or ERK1/2, which will enhance the HIF-1 alpha pathway as well. ${ }^{14}$

Thus, a drug that can control the mTOR-HIF-1 alphaVEGF pathway in breast cancer will be a very promising therapy for breast cancer. ${ }^{15-17}$ Many drugs only target one component of the mTOR-HIF-1 alpha-VEGF signaling axis, however, we need a drug with ability to control all of them with high efficiency and potency. Vandetanib is a medullar thyroid cancer drug approved in 2011. ${ }^{18-20}$ VEGF receptor-2, EGFR, and RET tyrosine kinases are targets of vandetanib. ${ }^{18-20}$ This new drug has been experimented to treat other cancers. MAPK, mTOR, and AKT were inhibited by vandetanib in medullary thyroid cancer. Rho-JNK and autophagy pathways were inhibited by this drug as well. ${ }^{21}$ Vandetanib can inhibit mTOR and VEGF signaling. Current studies have not yet shown that vandetanib target HIF-1 alpha. Therefore, the inhibition of mTOR, HIF-1 alpha, and VEGF signaling pathway is rare in literature. Here, we hypothesized that vandetanib may inhibit breast cancer growth via antiangiogenesis. We found vandetanib can target mTOR-HIF-1 alpha-VEGF signaling axis in breast cancer cells. Therefore, vandetanib will be a very promising drug for breast cancer since it has been proven to treat thyroid cancer recently.

\section{Materials and methods}

\section{Cell culture}

MDA-MB-231 and MCF-7 (ATCC, Manassas, VA, USA) were cultured in DMEM (Gibco, Billings, MT, USA) medium supplied with other factors in an incubator (SANYO, Osaka, Japan) at $37^{\circ} \mathrm{C}$ with $5 \% \mathrm{CO}_{2}$. Changed medium or passage every 2-3 days. Healthy cells were used for cell growth, apoptosis, cell cycle analysis, gene expression, Western blot, and ELISA. The cells were divided into three groups: vandetanib group: cells were treated with $100 \mu \mathrm{L}$ vandetanib (50 $\mathrm{g} / \mathrm{mL}$, MedChemExpress, Princeton, NJ, USA); negative control (NC) group: cells were treated with
$100 \mu \mathrm{L}$ dimethyl sulfoxide (DMSO); and control group: cells were not treated with anything.

\section{MTT assay}

About $5 \times 10^{3}$ breast cancer cells were seeded in each well of 96-well plates and cultured for 24 hours. Vandetanib was used to treat the cells at a concentration of $50 \mu \mathrm{g} / \mathrm{mL}$ for 12 hours. Then, $20 \mu \mathrm{L}$ of MTT solution $(5 \mathrm{mg} / \mathrm{mL})$ was added to culture and incubated for 4 hours. After removing culture medium, $150 \mu \mathrm{L}$ DMSO was added to each well to dissolve the crystal by shaking in dark for 15 minutes. The $490 \mathrm{~nm}$ absorbance was measured with a microplate reader (Bio-Rad Laboratories, Hercules, CA, USA). Growth rate $(\%)=$ $\left(\mathrm{OD}_{\text {Vandetanib }}-\mathrm{OD}_{\text {Solution }}\right) /\left(\mathrm{OD}_{\text {Control }}-\mathrm{OD}_{\text {Solution }}\right) \times 100 \%$.

\section{Measure of apoptosis by flow cytometry}

About $2 \times 10^{5}$ breast cancer cells were seeded in each well of 6-well plates and cultured overnight at $37^{\circ} \mathrm{C}$. Vandetanib was used to treat the cells at a concentration of $50 \mu \mathrm{g} / \mathrm{mL}$ for 24 hours. The cells were collected after digested with trypsin and centrifuged at 1,000 rpm for 5 minutes. After washing with cold PBS (Gibco) twice, $2 \times 10^{5}$ breast cancer cells were suspended in $100 \mu \mathrm{L}$ buffer with $5 \mu \mathrm{L}$ Annexin-V fluorescein isothiocyanate and $5 \mu \mathrm{L}$ propidium iodide (PI) (Oncogene, Manhasset, NY, USA). After incubated for 15 minutes at room temperature, cells were diluted in $400 \mu \mathrm{L}$ buffer for flow cytometry (Gallios, Beckman Coulter, Inc, Brea, CA, USA).

\section{Cell cycle analysis}

Breast cancer cells were seeded in each well of 6-well plates and cultured till reached $70 \%-80 \%$ of confluence and vandetanib was added to a concentration of $50 \mu \mathrm{g} / \mathrm{mL}$ for 24 hours. Similarly, cells were collected after digested by trypsin and centrifuged at 1,000 rpm for 5 minutes. After washing with cold PBS twice, centrifuged at 1,000 rpm for 5 minutes, then the cells were fixed with $70 \% \mathrm{EtoH}$ at $4^{\circ} \mathrm{C}$ for 12 hours. Cells collected were centrifuged at 15,000 rpm for 5 minutes and washed with PBS once. The cells were suspended in $400 \mu \mathrm{L}$ buffer containing PI and RNase (BD pharmingen, San Diego, CA, USA) and incubated for 15 minutes at room temperature. Then, the cells were analyzed by flow cytometry (Gallios, Beckman Coulter, Inc).

\section{Detection of VEGF in supernatant by ELISA}

After treatment with vandetanib for 24 hours, the supernatants of multiple wells were collected by centrifugation at 1,000 rpm for 5 minutes. ELISA kit (ABIN2506955, BioAim 
Scientific, Toronto, Canada) was used to detect VEGF concentration by measuring OD 450 with a microplate reader (RT-6100, Lei Du).

\section{Quantitative real-time (qRT)-PCR}

TRIzol kit (Invitrogen, Grand Island, NY, USA) was used to isolate total RNA (OD260/OD280=1.8-2.0) Superscript III Reverse Transcriptase (Thermo Fisher Scientific, Waltham, MA, USA) was used to reverse cDNA. Master ep realplex2 (Eppendorf, Hamburg, Germany) was used for qRT-PCR. The program was as follows: $94^{\circ} \mathrm{C}$ for 3 minutes, $94^{\circ} \mathrm{C}$ for 45 seconds, and $58^{\circ} \mathrm{C}$ for 45 seconds ( 30 cycles). The data were collected and analyzed by the method of $2^{-\Delta \Delta \mathrm{Ct}}$, and the relative expression level was calculated by using $\beta$-actin as the internal reference. The primers were synthesized by Sangon Biotech, Shanghai, China, and the sequences are shown in Table 1.

\section{Western blot}

Protein concentration of cellular extracts was determined by bicinchoninic acid method. Then, protein samples $(4 \mu \mathrm{L})$ were loaded on $10 \%$ SDS-PAGE for electrophoresis. Primary antibodies, including mTOR (1:1,000, ABIN2855088, Antibodies-online, Aachen, Germany), HIF-1 alpha (1:500, ab216842, Abcam, Cambridge, UK), VEGF (1:1,000, ABIN964761, Antibodies-online), $\beta$-actin (1:1,000, ABIN2854709, Antibodies-online) and secondary antibody IgG-horseradish peroxidase (HRP) (1:1,000, \#7074, Cell Signaling Technology, Beverly, MA, USA) were used for Western blot. Elecsys-2010 (Roche Professional Diagnostics, Basel, Switzerland) were used for data collection. ImageJ (National Institutes of Health [NIH]) software was used to quantify gray level of all the bands from image, and $\beta$-actin band was used to normalize data.

\section{HUVECs culture}

Conditional medium was made of supernatant of breast cancer cells medium, DMEM, and FBS at a ratio of 4:5:1,

Table I Primers used in gene expression analysis

\begin{tabular}{l|l}
\hline Name & Sequence \\
\hline mTOR & $\begin{array}{l}\text { Forward: 5'-GCTAGGTGCATTGACATACAACA-3' } \\
\text { Reverse: 5'-AGTGCTAGTTCACAGATAATGGC-3' }\end{array}$ \\
HIF-I $\alpha$ & $\begin{array}{l}\text { Forward: 5'-GATGTAATGCTCCCCTCAC-3' } \\
\text { Reverse: 5'-GCTGGAATACTGTAACTGTGC-3' } \\
\text { VEGF }\end{array}$ \\
$\beta$-actin & $\begin{array}{l}\text { Forward: 5'-GGCTGGCAACATAACAGAGAA-3' 5'-CCCCACATCTATACACACCTCC-3' } \\
\text { Forward: 5'-GTTTTCCTCATCGAGGCATCTG-3' } \\
\text { Reverse: 5'-CCAAAATGACTGGTTAGCTGGTA-3' }\end{array}$ \\
\hline
\end{tabular}

Abbreviations: $m T O R$, mechanistic target of rapamycin; VEGF, vascular endothelial growth factor. respectively. HUVECs purchased by ATCC were cultured in DMEM with 10\% FBS, $2 \mathrm{mM}$ Gln, $100 \mathrm{IU} / \mathrm{mL}$ penicillin, $100 \mu \mathrm{g} / \mathrm{mL}$ streptomycin, and 1\% non-essential amino acids (Gibco). To further address VEGF on vandetanib-induced antiangiogenesis effect, we used NC group (100 $\mu \mathrm{L}$ DMSO), ZD6474 group (100 $\mu \mathrm{L}$ ZD6474 [50 $\mu \mathrm{g} / \mathrm{mL}])$, VEGF group (100 $\mu \mathrm{L}$ DMSO and $100 \mu \mathrm{L}$ VEGF [Santa Cruz Biotechnology Inc., Santa Cruz, CA, USA] [10 ng/mL]), ZD6474+ VEGF group $(100 \mu \mathrm{L} Z \mathrm{ZD} 6474[50 \mu \mathrm{g} / \mathrm{mL}]$ and $100 \mu \mathrm{L}$ VEGF [10 ng/mL]), DMSO + anti-VEGF group (100 $\mu \mathrm{L}$ DMSO and $100 \mu \mathrm{L}$ anti-VEGF [Santa Cruz] [10 ng/mL]), anti-VEGF + ZD6474 group $(100 \mu \mathrm{L}$ ZD6474 [50 $\mu \mathrm{g} / \mathrm{mL}]$ and $100 \mu \mathrm{L}$ anti-VEGF [10 ng/mL]) and control group.

\section{Wound healing experiment}

HUVECs were seeded in 24-well plates and grew to $100 \%$ confluency. Then the cells were scratched by a tip $10 \mu \mathrm{L}$ pippet tip. The cells were washed three times with PBS and then cultured in conditional medium for 24 hours. Microscope (Olympus, Tokyo, Japan) was used to take pictures for analysis.

\section{Transwell and invasion assays}

Matrigel (BD Biosciences, San Jose, CA, USA) was mixed with ice-cold DMEM culture medium in a ratio of $1: 1$ and $300 \mu \mathrm{L}$ of this mixture was plated in the upper well of transwell (Corning Life Sciences, Corning, NY, USA). Then, $100 \mu \mathrm{L}$ of HUVECs $\left(5 \times 10^{4}\right)$ was seeded. The bottom well contained $500 \mu \mathrm{L}$ of conditional culture medium. The transwell was incubated in an incubator under $5 \% \mathrm{CO}_{2}$ and $37^{\circ} \mathrm{C}$ for 72 hours. The upper well was washed with PBS twice and fixed with $5 \%$ glutamyhyde at $4^{\circ} \mathrm{C}$. After washing with $\mathrm{PBS}$ twice, $0.1 \%$ crystal violet was used to stain for 30 minutes. After washed with PBS for two times, microscope (Olympus, Tokyo, Japan) was used to take pictures.

\section{Tubular formation experiment}

Matrigel was added to 96 wells and stayed at $37^{\circ} \mathrm{C}$ for 1 hour. Then, $4 \times 10^{4}$ HUVECs were seeded in the 96 well with conditional medium to grow under $5 \% \mathrm{CO}_{2}$ and $37^{\circ} \mathrm{C}$ for 48 hours. Then took pictures with microscope (CKX40, Olympus, Brooklyn, NY, USA; Jenoptik ProgRes Camera, Thuringia, Germany) for data analysis.

\section{Construction of xenograft tumor animal models}

Animal experiments follow NIH guidelines (NIH Pub. No 85-23, revised 1996) and have been approved by the Animal Protection and Use Committee of Tengzhou Central People's Hospital. 
Eighteen 4-week-old Balb/c nude mice $(20 \pm 2 \mathrm{~g})$ were selected and purchased from Jinan Pengyue Laboratory Animal Breeding Co., Ltd. under the license number SCXK (Lu) 20140007. They were randomly divided into three groups $(\mathrm{n}=6)$ : blank control group (normal saline), DMSO group, and ZD6474 group. Then, $0.2 \mathrm{~mL}$ MCF-7 cell suspension $\left(1 \times 10^{6}\right.$ cells $)$ was subcutaneously injected into the left axilla of nude mice. The tumor volume was measured every 3 days, and the average volume of tumor was calculated as follows: Volume $\left(\mathrm{mm}^{3}\right)=\left(\right.$ length $\times$ width $\left.^{2}\right) / 2$. Six weeks later, the nude mice were anesthetized with $0.3 \%$ sodium pentobarbital (45 mg/kg), followed by the cervical vertebra dislocated and the tumor was removed and weighed, then stored in $4 \%$ paraformaldehyde.

\section{Detection of microvessel density (MVD)}

Paraffin sections of breast tumors in nude mice were taken. The sections were $5 \mu \mathrm{m}$ thick, dewaxed with xylene, and repaired with antigen of high temperature and high pressure tissue. CD34 is located in the cytoplasm of vascular endothelial cells and is brownish yellow. CD34 immunostaining specimens were used to label the microvasculature, and brown-yellow granules in the cytoplasm of vascular endothelial cells were used as positive criteria. MVD counting: the CD34 positive vessels were observed under a low power microscope (40 times), the areas with dense coloring cells were selected, and the colored vessels were identified under microscope (200 X). Five non-repeated fields of view were selected to count under microscope (200 X). The average value is the MVD value of the slice. The aforementioned immunohistochemical staining results were determined by three technical personnel with many years of experience in pathological diagnosis.

\section{Tumor cell apoptosis assay}

Fluorescein-labeled dUBP can connect to the $3^{\prime}$-OH terminal of DNA which is broken in apoptotic cells due to the action of deoxyribonucleotide terminal transferase. According to the instructions of TUNEL cell apoptosis detection kit, the breakage of nuclear DNA during apoptosis can be detected. Five non-repeated visual fields were randomly selected for counting and statistical analysis. The apoptotic index was calculated by counting the percentage of positive cells in the total number of cells.

\section{Immunohistochemistry}

Paraffin embedding was proceeded to dewaxing and hydration $(4 \mu \mathrm{m})$, inactivation of endogenous peroxidase was performed, followed by electric furnace heating repair antigen. BSA sealing solution 5\% was added and incubated at room temperature for 20 minutes. Anti mTOR (1:50, ABIN 2855088, Antibodies-online), HIF-1 alpha (1:100, ab216842, Abcam), and VEGF (1:100, ABIN 964761, Antibodies-online), were added at $4{ }^{\circ} \mathrm{C}$ overnight. Then second antibodies (1:800, 85-9043, HRP, Broad Spectrum) were added with State Agricultural and Biotechnology Centre incubation. Diaminobenzidine colored was performed, followed by hematoxylin light dyeing. Then the sections were detected under microscopy (Olympus BX51, Olympus).

\section{Statistics}

SPSS 19.0 was used to analyze data and all data were expressed as mean $+\mathrm{SD}$. Multiple comparisons were evaluated by ANOVA followed by the least significant difference test and $P<0.05$ was considered significant.

\section{Results \\ Vandetanib modulated the cellular behavior of breast cancer cells}

As shown in Figure 1, vandetanib can significantly reduce cell proliferation compared with control and $\mathrm{NC}$ groups. About 12 hours after treatment, cell viability decreased about half by MTT assay. We hypothesize that apoptosis might be the cause of this effect. Therefore, Annexin V/PI staining was performed for three groups at 24 hours after treatment. Flow cytometry assay showed that vandetanib group produced about four folds more Annexin V/PI double positive cells compared with other two groups. Cell cycle analysis was performed. As to $\mathrm{G} 2 / \mathrm{M}$ phage, there was significant reduction compared with control group $(P<0.05)$. As to $\mathrm{G} 0 / \mathrm{G} 1$ phage, the percentage was improved $(P>0.05)$. Thus, vandetanib inhibited the proliferation of breast cancer cells through regulating G2/M phage and subsequently contributed to growth inhibition.

\section{Vandetanib played roles in mTOR, HIF-I alpha, and VEGF expression}

To further dissect molecular pathway involved in vandetanibinduced growth inhibition, we isolated mRNA and cellular extracts for gene expression and Western blot analysis, respectively. Expression of mTOR, HIF-1 alpha, and VEGF mRNA were all scientifically decreased about four folds compared with control and $\mathrm{NC}$ groups (Figure 2A and C). Western blot further confirmed the finding at protein expression level (Figure 2B and D). The mTOR and HIF-1 alpha protein decreased about five folds while the VEGF decreased 
A

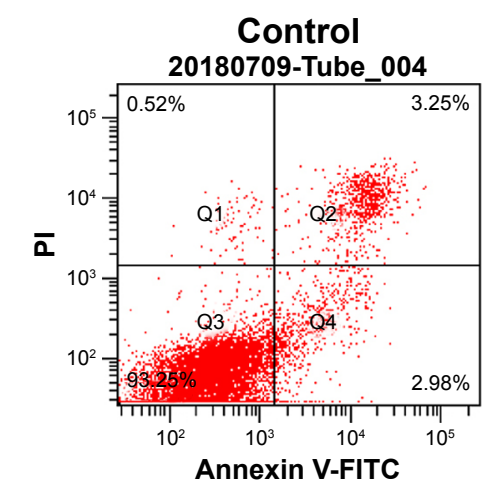

B

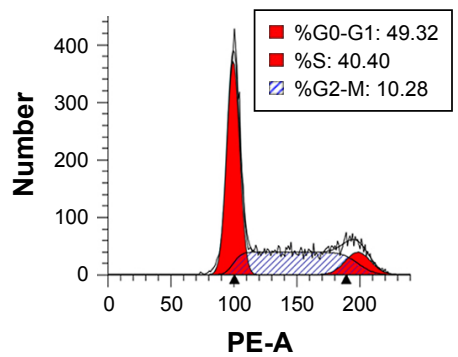

C

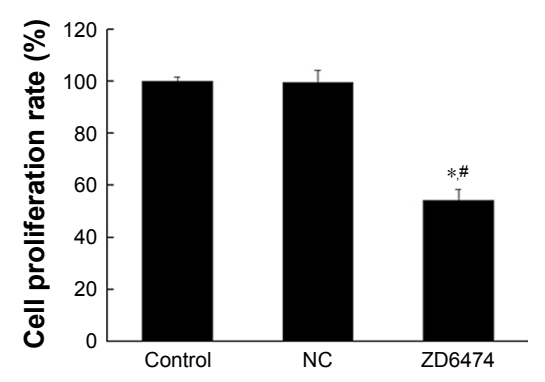

D
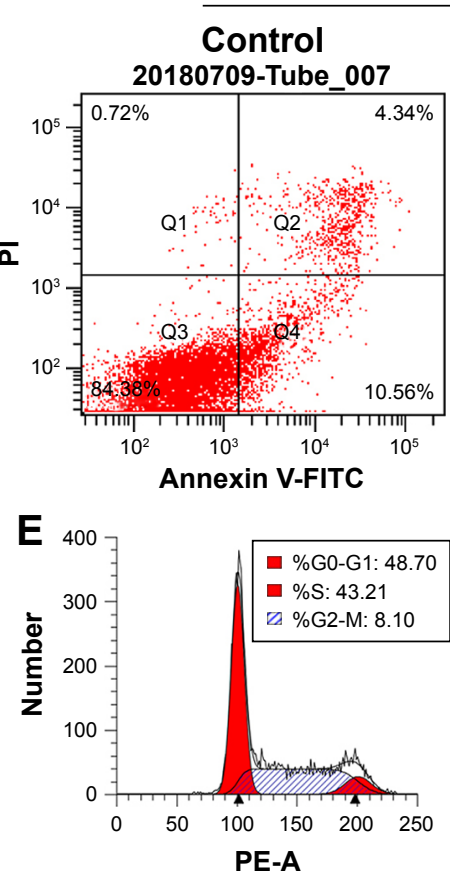

MDA-MB-231
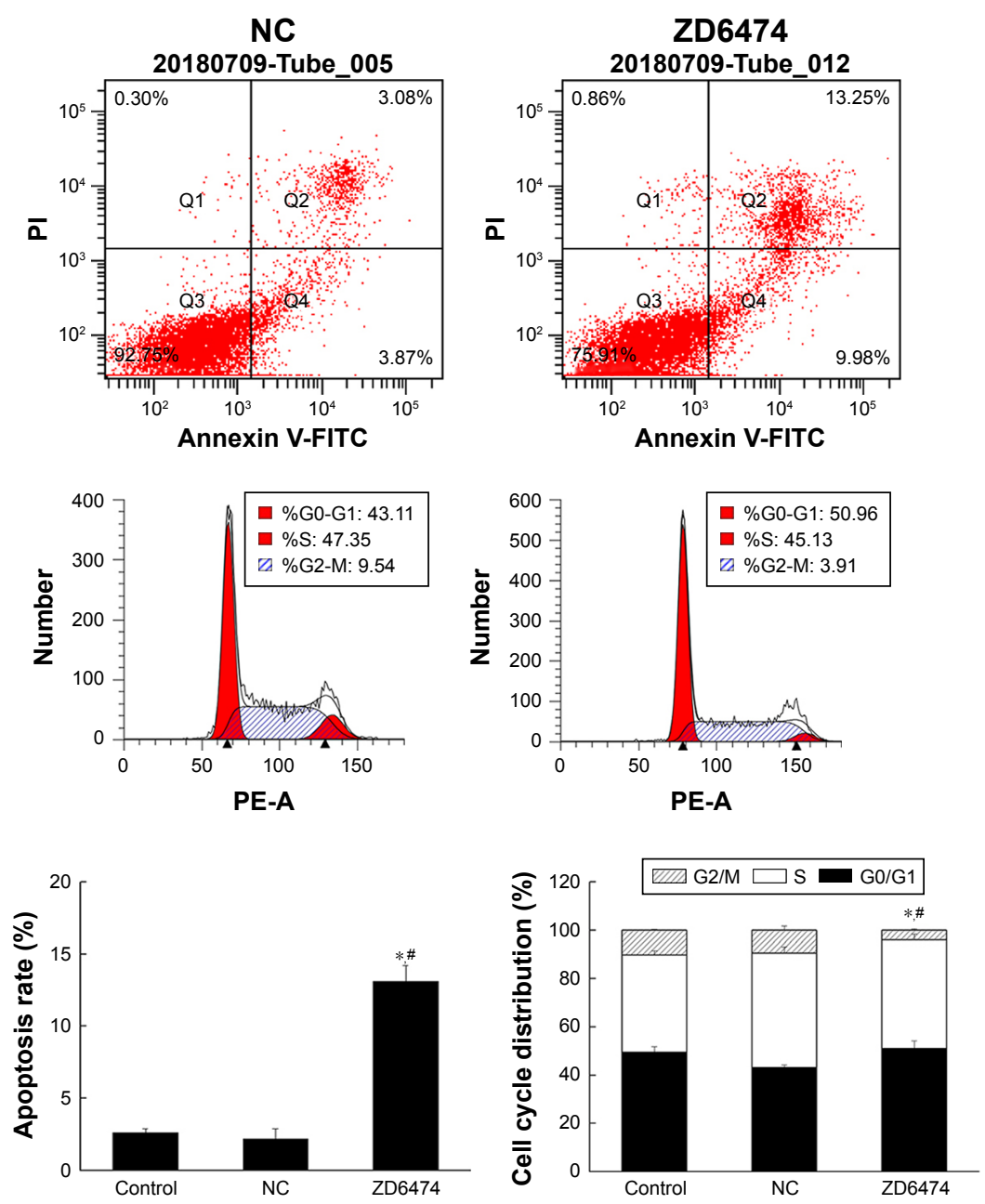

MCF-7

NC

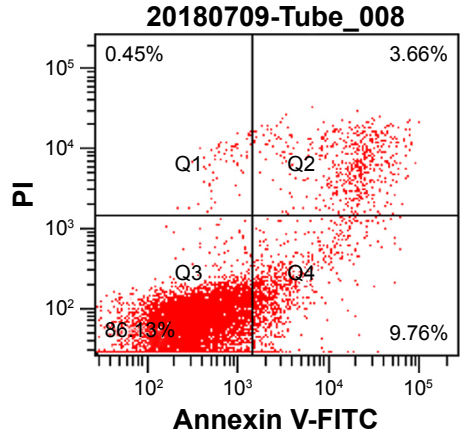

ZD6474
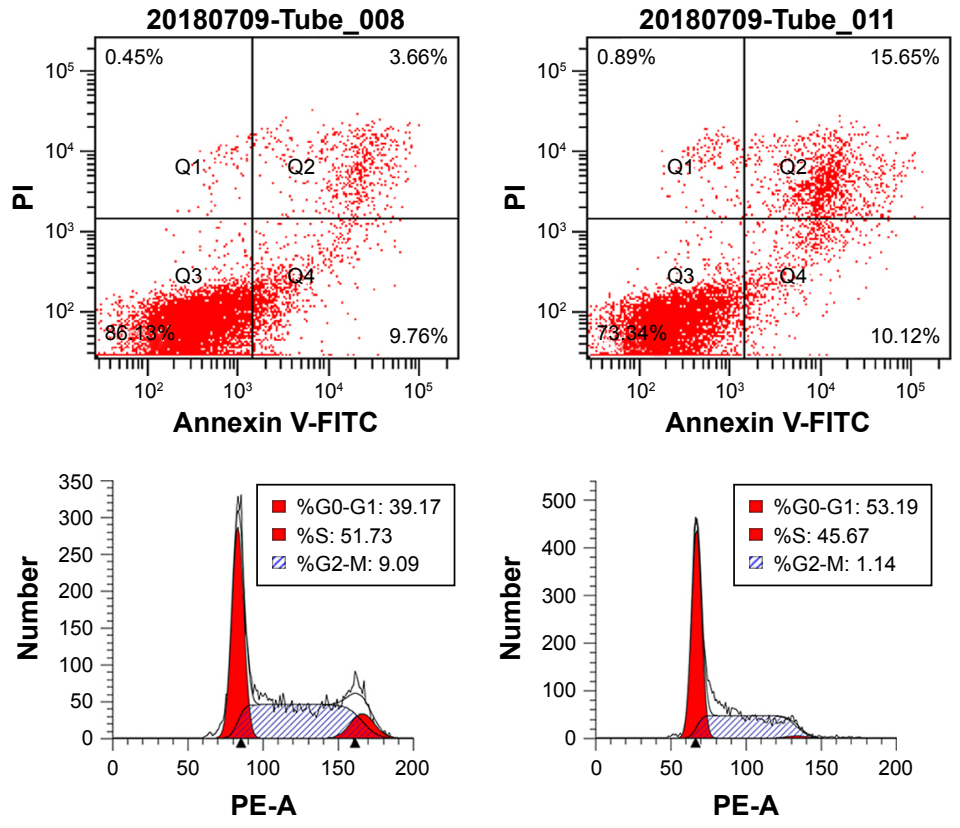

Figure I (Continued) 

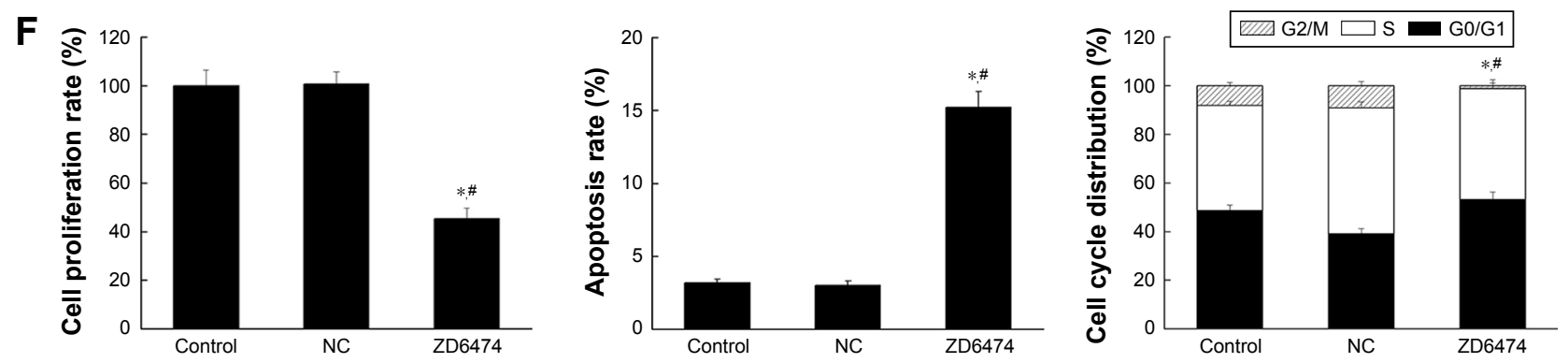

Figure I Vandetanib induced growth inhibition, apoptosis, and cell cycle arrest.

Notes: (A) MDA-MB-23I apoptosis under vandetanib treatment was measured by flow cytometry with Annexin V/PI staining. (B) MDA-MB-23I cell cycle analysis upon vandetanib treatment by flow cytometry with PI staining. (C) MDA-MB-23 I growth inhibition, quantification of apoptosis and quantification of cell cycle induced by vandetanib. (D) MCF-7 apoptosis under vandetanib treatment was measured by flow cytometry with Annexin V/PI staining. (E) MCF-7 cell cycle analysis upon vandetanib treatment by flow cytometry with PI staining. (F) MCF-7 growth inhibition, quantification of apoptosis, and quantification of cell cycle induced by vandetanib. $* P<0.05$ (compared with control) or ${ }^{\#}<0.05$ (compared with $\mathrm{NC}$ ) was considered significant.

Abbreviations: NC, negative control; Pl, propidium iodide.

A

B
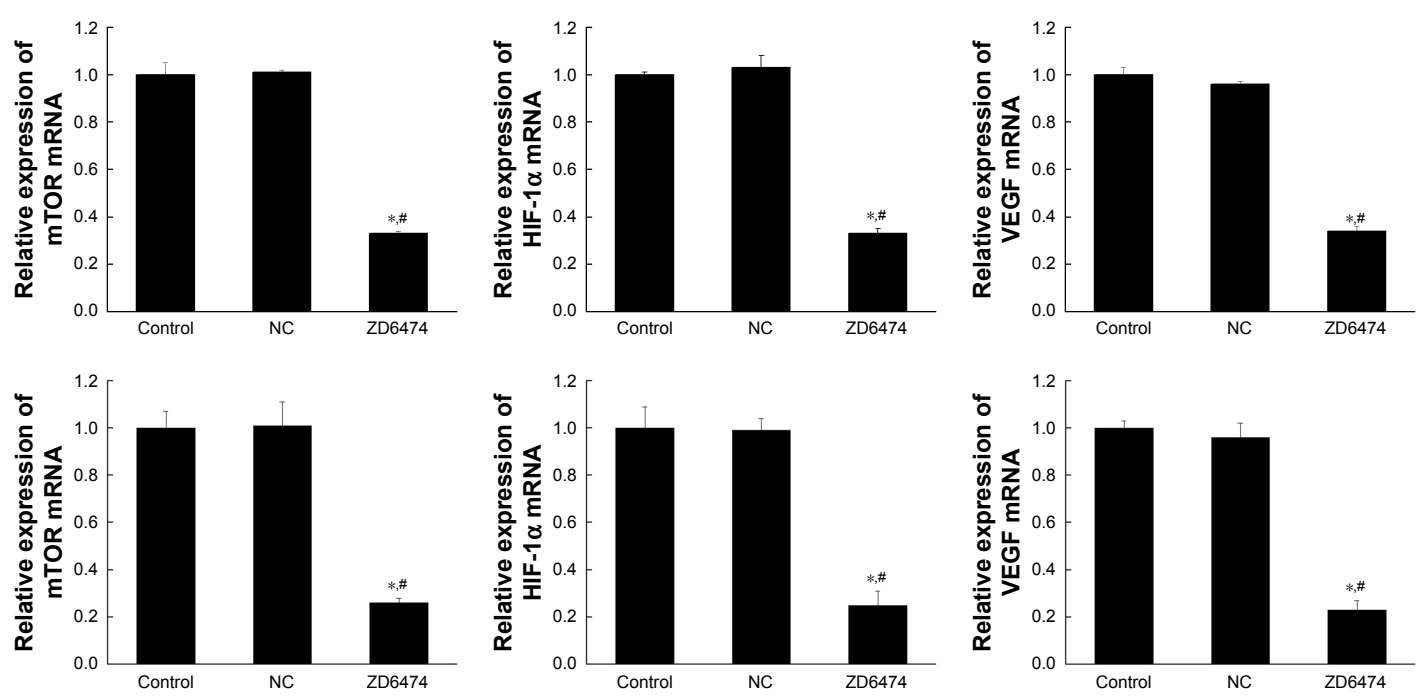

C
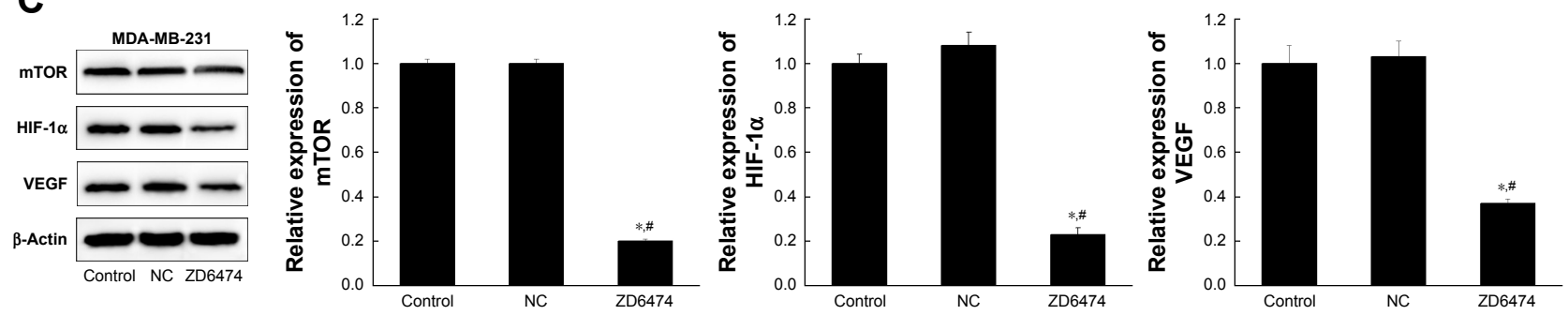

D
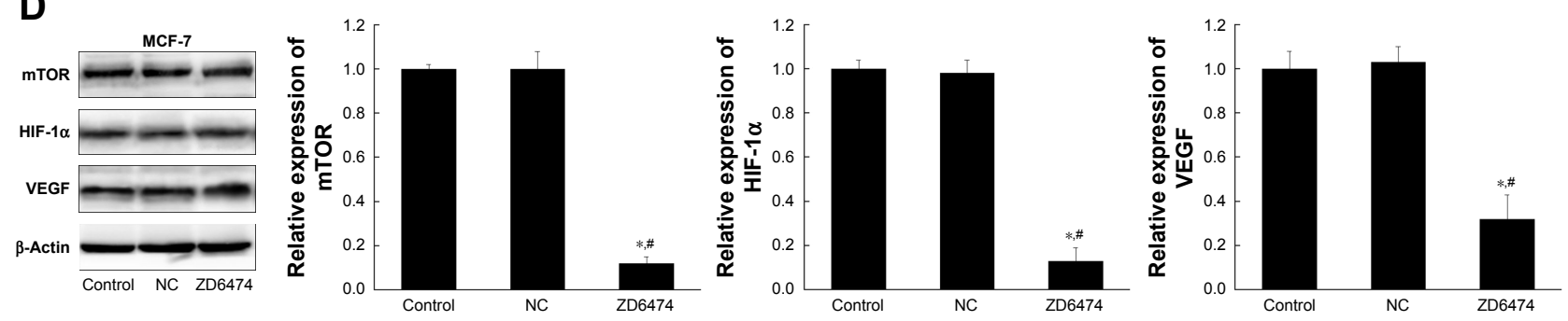

Figure 2 Vandetanib on gene expression and protein expression in breast cancer cells.

Notes: (A) MDA-MB-23I gene expression analysis upon vandetanib by qRT-PCR for mTOR, HIF-I alpha, and VEGF. (B) MDA-MB-23I Western blot analysis of protein expression of mTOR, HIF-I alpha, and VEGF under vandetanib treatment in MDA-MB-23I cells. (C) MCF-7 gene expression analysis upon vandetanib by qRT-PCR for mTOR, HIF-I alpha, and VEGF. (D) MCF-7 Western blot analysis of protein expression of mTOR, HIF-I alpha, and VEGF under vandetanib treatment in MDA-MB-23I cells. $* P<0.05$ (compared with control) or ${ }^{\#}<<0.05$ (compared with $\mathrm{NC}$ ) was considered significant.

Abbreviations: HIF, hypoxia-inducible factor; mTOR, mechanistic target of rapamycin; NC, negative control; qRT, quantitative real-time; VEGF, vascular endothelial growth factor. 
about three folds compared with control and NC groups $(P<0.05)$.

\section{VEGF level in the supernatant of culture was analyzed by ELISA}

As shown in Figure 3, the VEGF level decreased about $35.0 \%$ upon vandetanib treatment $(P<0.05)$.

\section{HUVEC cells were used for angiogenesis analysis upon vandetanib treatment}

Because the effect of ZD6474 on MDA-MB-231 and MCF-7 was basically consistent, the tumor condition medium cultured by MCF-7 was used for subsequent experiments. VEGF could induce significant wound healing effect compared with control and NC groups (Figure 4A). But vandetanib significantly inhibited wound healing in both with or without VEGF treatment compared with $\mathrm{NC}$ or control group $(P<0.05)$. Similarly, transwell invasion of HUVEC increased upon VEGF treatment and decreased upon anti-VEGF treatment (Figure 4B). However, vandetanib reduced this behavior for groups with or without VEGF treatment compared with NC or VEGF group. Tubular formation ability was higher when VEGF used to treat the HUVEC (Figure 4C). Vandetanib significantly decreased the tubular formation under conditions with or without VEGF. All aforementioned results demonstrated that VEGF can stimulate angiogenesis; however, vandetanib inhibited all the angiogenesis behavior.

\section{Vandetanib inhibited the growth of breast tumor}

In order to investigate whether vandetanib regulates breast cancer carcinogenesis in vivo, we investigated vandetanibtreated breast tumor growth in vivo, using a subcutaneous xenograft tumor model in mice. We found that the tumor size (Figure 5A and B) and tumor weight (Figure 5C) of vandetanib-treated breast cancer xenografts were significantly smaller than that of vandetanib-untreated tumors. Additionally, the survival rates of these mice indicated that vandetanib-treated breast cancer mice had significantly higher survival rates than vandetanib-untreated breast cancer mice $(P<0.5$, Figure 5D). These strongly support that vandetanib inhibited the growth of breast tumor in vivo. Then the tumors were further investigated. The immunohistochemistry of tumors showed that vandetanib significantly inhibited the angiogenesis $(P<0.5$, Figure 6$)$. The TUNEL analysis of tumors showed that the cell apoptosis was promoted significantly $(P<0.5)$ by vandetanib (Figure 7$)$. Furthermore, we found that mTOR, HIF-1 alpha, and VEGF expression were significantly inhibited by vandetanib (Figure 8 ).

\section{Discussion}

Neo-angiogenesis is very important for tumor growth and metastasis. The pathogenesis and progression of breast cancer is largely due to VEGF and its signaling pathway. ${ }^{23,24}$ When tumor cell number increases, the demand for oxygen is not met by the existing vascular system. Therefore, hypoxia occurs and induces a variety of gene expression modulation to promote HIF-1 alpha stability and signaling., ${ }^{8,22}$ Gene $m T O R$ is the upstream regulator of HIF-1 alpha. HIF-1 alpha is a transcriptional factor which can active many genes. On the other hand, some will promote angiogenesis to get more circulation in the neoplasia tissue. With these genes, tumor cells will keep progressing. . $^{14,16}$

Our study shows that vandetanib can regulate all the angiogenesis genes, which will further impact the survival, invasion, and neo-angiogenesis for breast cancer cell lines.
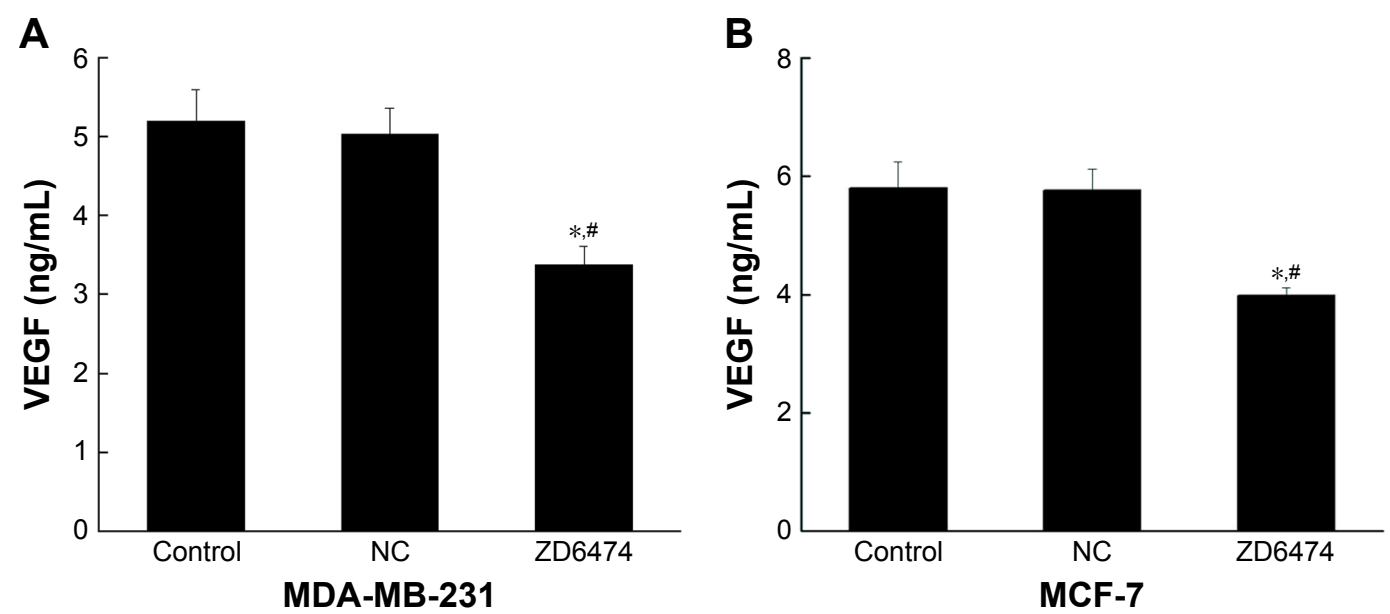

Figure 3 Vandetanib affecting the secretion of VEGF in breast cancer cells culture supernatant.

Notes: (A) MDA-MB-23I. (B) MCF-7. $* P<0.05$ (compared with control) or $\# \mathbb{P}<0.05$ (compared with NC) was considered significant.

Abbreviations: NC, negative control; VEGF, vascular endothelial growth factor. 

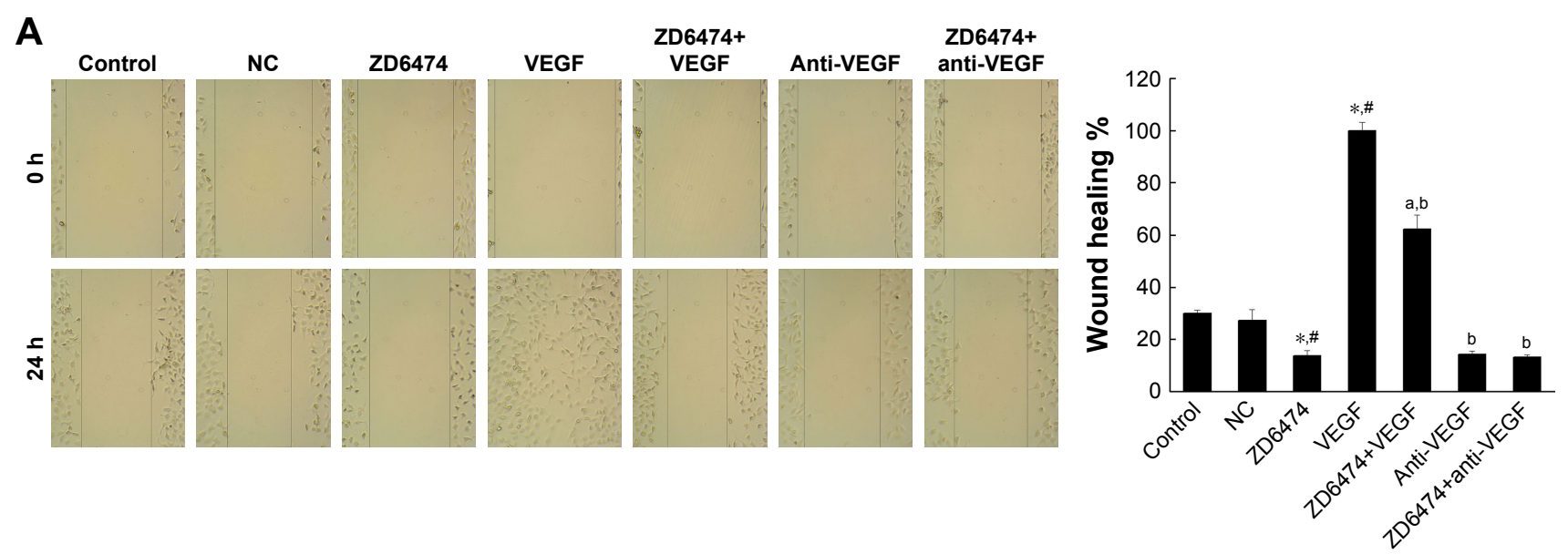

\section{B}
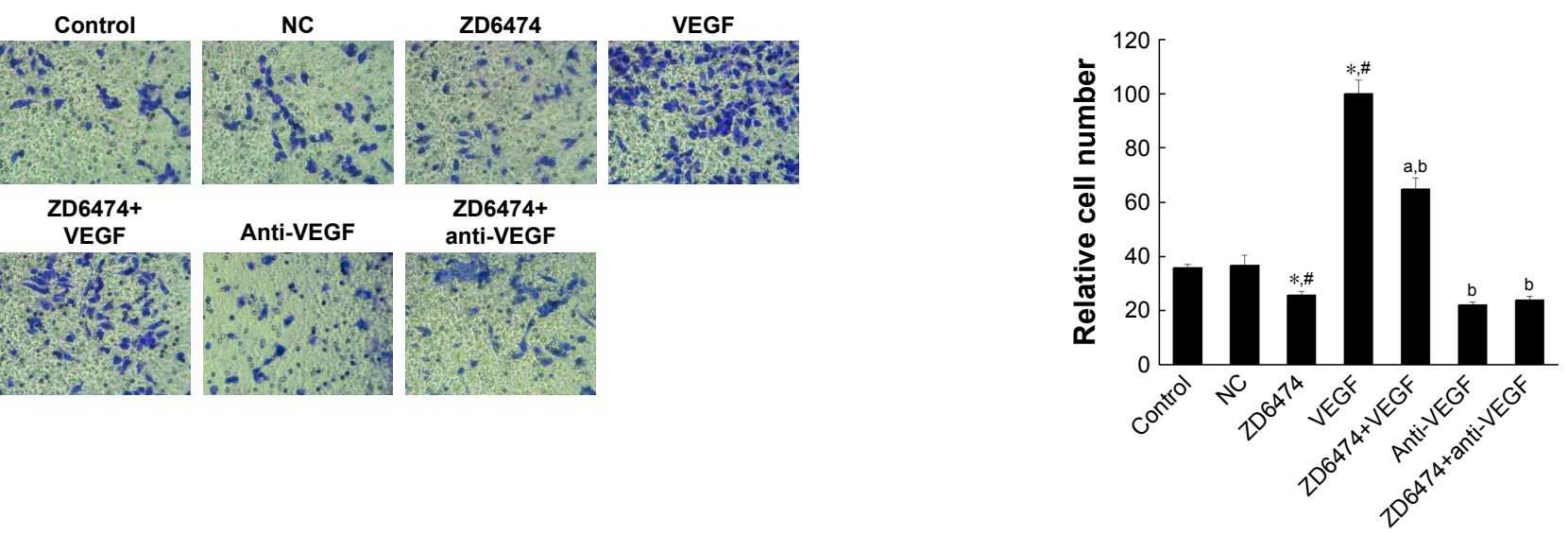

C
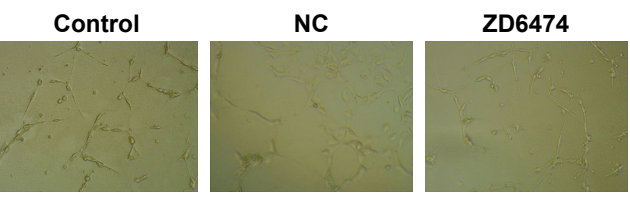

VEGF
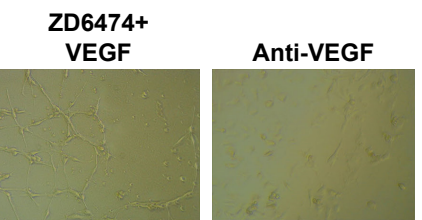

ZD6474+ anti-VEGF
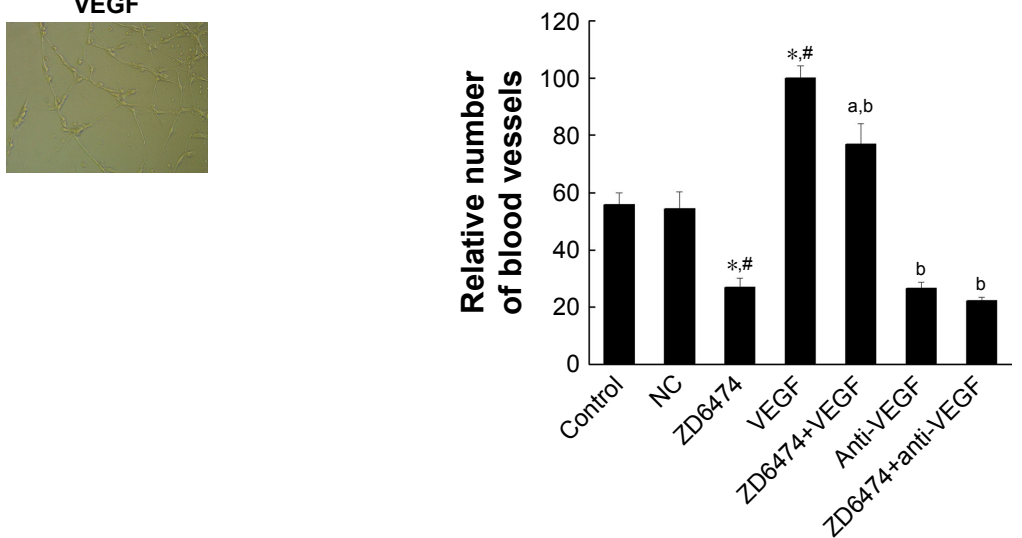

Figure 4 Cancerous cellular behavior changed for HUVECs upon vandetanib treatment.

Notes: (A) Wound healing experiment under vandetanib treatment and quantification data. (B) Transwell invasion assay of HUVECs upon vandetanib treatment and quantification data. (C) HUVECs tubular formation ability upon vandetanib treatment and quantification data. $* P<0.05$ (compared with control) or $\# P<0.05$ (compared with $\mathrm{NC}$ ) a $P<0.05$ (compared with vandetanib) or ${ }^{b} P<0.05$ (compared with VEGF) was considered significant. Magnification $\times 200$.

Abbreviations: NC, negative control; VEGF, vascular endothelial growth factor.

The controlling of all the related signaling molecules confer this treatment more robust efficacy and effectiveness. We will further test this medicine in animal models to validate what we found in this study. In addition, some other related researches about vanetanib on clinical trial have demonstrated the promising future of vandetanib for breast cancer. ${ }^{18,25}$
Studies showed that vandetanib is a promising treatment approach for breast cancer or even advanced breast cancer. It can be used alone or combined with other different methods. However, the mechanism is still unclear, and the results were not very significant. Therefore, a better understanding of mechanism will help us to identify the best 


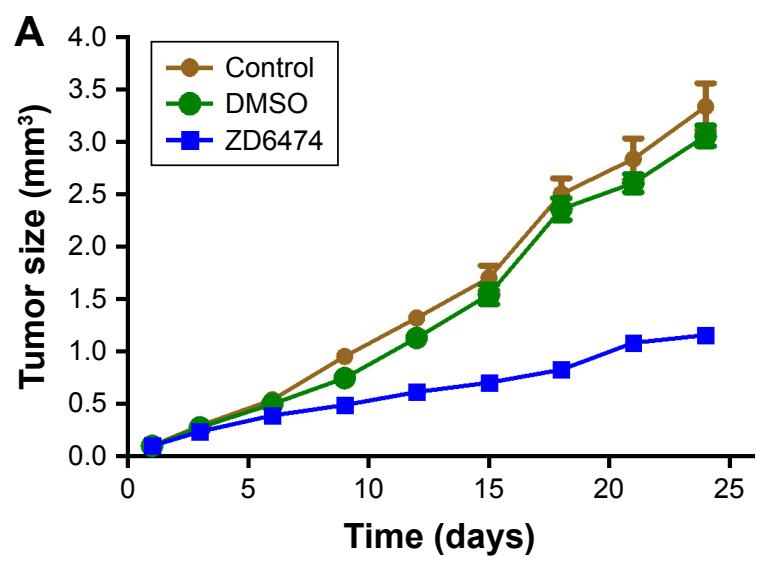

B
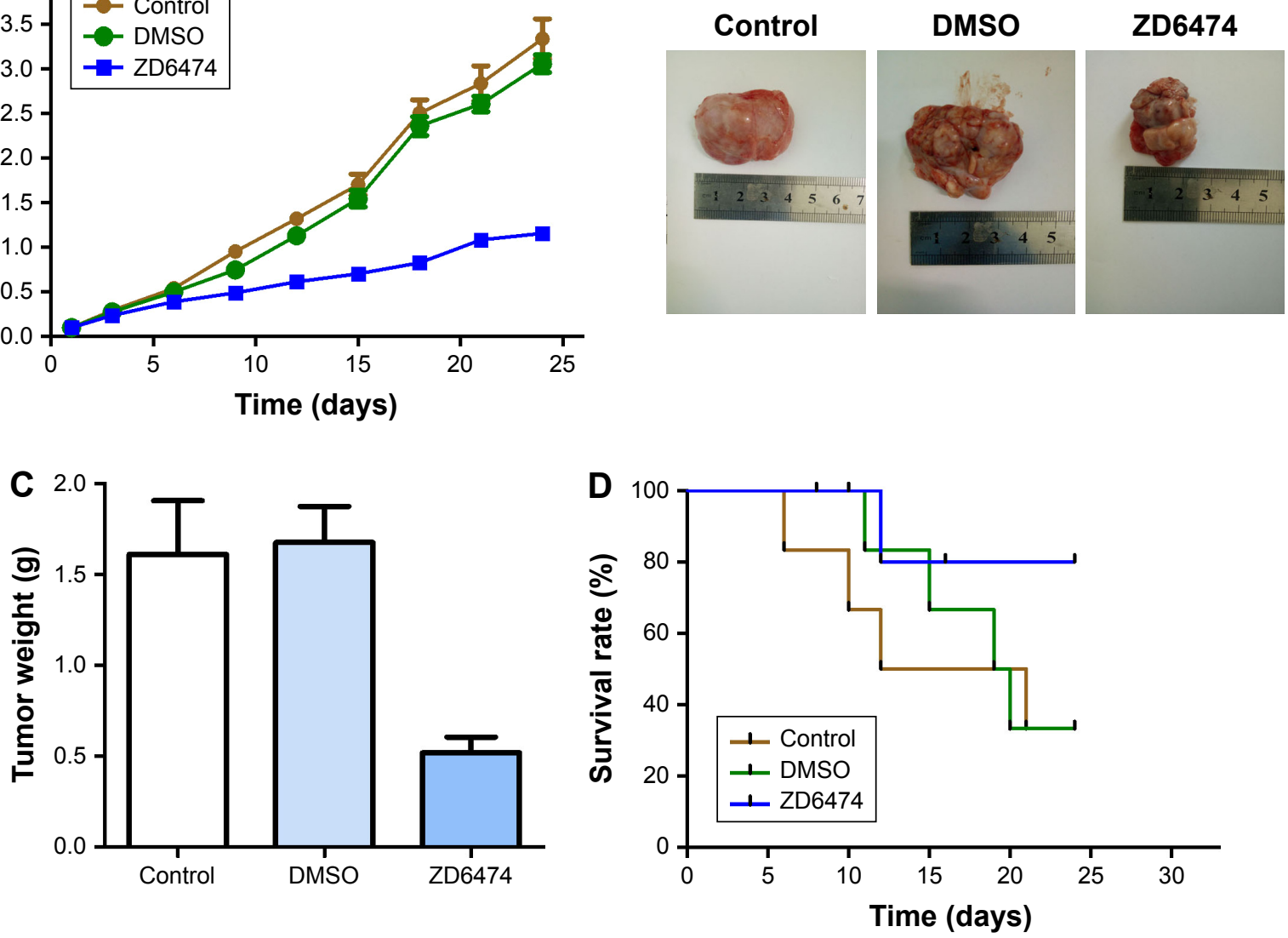

Figure 5 Vandetanib inhibited the growth of breast tumor in mice.

Notes: (A) Tumor size. (B) Tumor figure. (C) Tumor weight. (D) Survival rates. Abbreviation: DMSO, dimethyl sulfoxide.
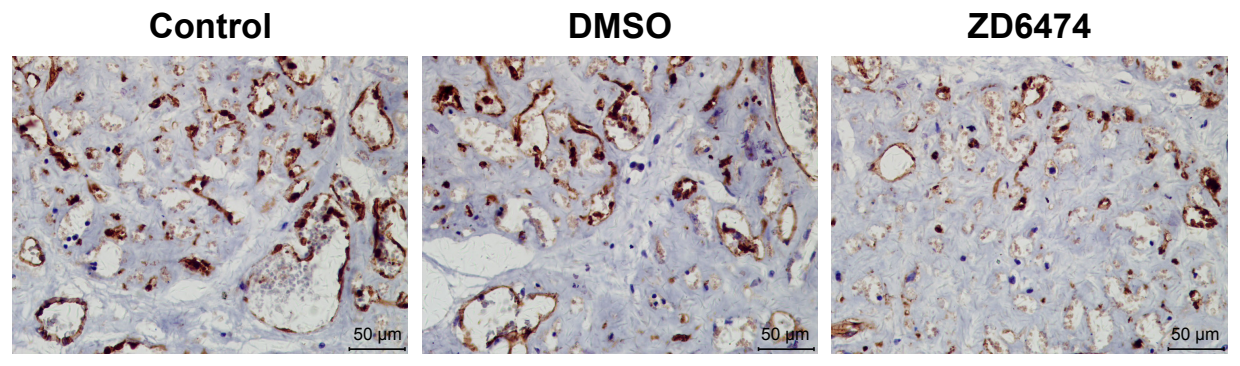

Figure 6 Vandetanib inhibited the angiogenesis in mice.

Note: Magnification $\times 400$.

Abbreviation: DMSO, dimethyl sulfoxide.
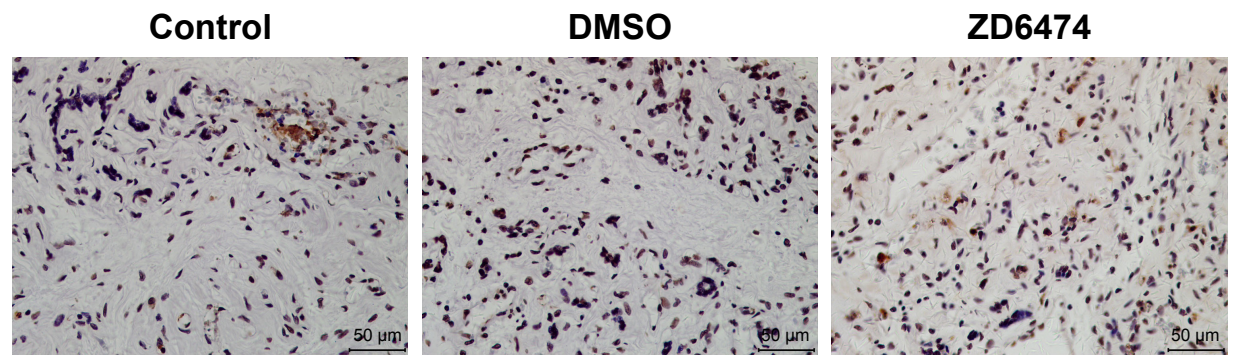

Figure 7 Vandetanib promoted the cell apoptosis in mice.

Note: Magnification $\times 200$.

Abbreviation: DMSO, dimethyl sulfoxide. 

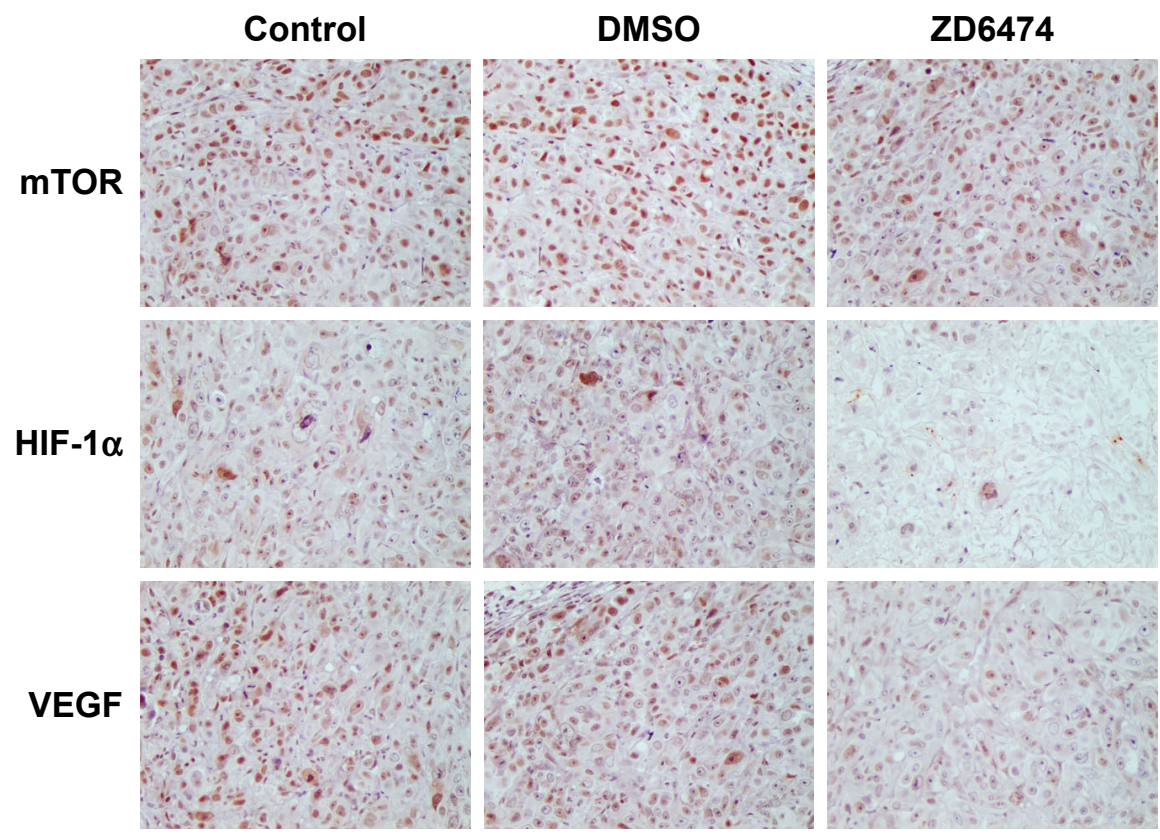

Figure 8 Vandetanib inhibited mTOR, HIF-I alpha, and VEGF expression.

Note: Magnification $\times 200$.

Abbreviations: DMSO, dimethyl sulfoxide; HIF, hypoxia-inducible factor; mTOR, mechanistic target of rapamycin; VEGF, vascular endothelial growth factor.

target for this new drug. Our study showed that vandetanib specifically targets mTOR, HIF-1 alpha, and VEGF signaling pathway. This will help us identify the best target for treatment. A cancer with upregulation of these genes will be the best target population. The combination with other effective treatment methods will benefit patients dramatically. In the future, we will continue to test our new finding in an in vitro model to validate our hypothesis that the synergistic targeting all the components of the pathway will be an alternative treatment of breast cancer. In addition, we will also explore the combination of vandetanib with other drugs, such as Tanshinone IIA and others, to develop personalized treatment for each type of breast cancer on an animal model. Eventually, we hope our finding will help clinical management of breast cancer and provide a new solution for certain population or new round of treatment.

\section{Conclusion}

Vandetanib can control angiogenesis of breast cancer in culture via mTOR, HIF 1 alpha, and VEGF signaling pathway.

\section{Acknowledgment}

This work was supported by Shandong Provincial Medical and Health Technology Development Project (No. 2016WS0627) and Young Teachers Research Support Fund of Jining Medical University (No. JY2016KJ041Y).

\section{Disclosure}

The authors report no conflicts of interest in this work.

\section{References}

1. Jeong SB, Im JH, Yoon JH, et al. Essential Role of Polo-like Kinase 1 (Plk1) Oncogene in tumor growth and metastasis of tamoxifen-resistant breast cancer. Mol Cancer Ther. 2018;17(4):825-837.

2. Kornblum N, Zhao F, Manola J, et al. Randomized phase II trial of fulvestrant plus everolimus or placebo in postmenopausal women with hormone receptor-positive, human epidermal growth factor receptor 2-negative metastatic breast cancer resistant to aromatase inhibitor therapy: results of PrE0102. J Clin Oncol. 2018;36(16):1556-1563.

3. Singh B, Sarli VN, Washburn LJ, Raythatha MR, Lucci A. A usable model of "decathlon winner" cancer cells in triple-negative breast cancer: survival of resistant cancer cells in quiescence. Oncotarget. 2018;9(13): 11071-11082.

4. Zhou H, Lv S, Zhang D, et al. A polypeptide based podophyllotoxin conjugate for the treatment of multi drug resistant breast cancer with enhanced efficiency and minimal toxicity. Acta Biomater. 2018;73: 388-399.

5. Ni Q, Zhang F, Zhang Y, et al. In situ shRNA synthesis on DNApolylactide nanoparticles to treat multidrug resistant breast cancer. Adv Mater. 2018;30(10).

6. Kopeć M, Abramczyk H. Angiogenesis - a crucial step in breast cancer growth, progression and dissemination by Raman imaging. Spectrochim Acta A Mol Biomol Spectrosc. 2018;198:338-345.

7. Dewangan J, Kaushik S, Rath SK, Balapure AK. Centchroman regulates breast cancer angiogenesis via inhibition of HIF-1 $\alpha /$ VEGFR2 signalling axis. Life Sci. 2018;193:9-19.

8. Liang H, Xiao J, Zhou Z, et al. Hypoxia induces miR-153 through the IRE1 $\alpha$-XBP1 pathway to fine tune the HIF1 $\alpha /$ VEGFA axis in breast cancer angiogenesis. Oncogene. 2018;37(15):1961-1975.

9. Pakravan K, Babashah S, Sadeghizadeh M, et al. MicroRNA-100 shuttled by mesenchymal stem cell-derived exosomes suppresses in vitro angiogenesis through modulating the mTOR/HIF-1 $\alpha / \mathrm{VEGF}$ signaling axis in breast cancer cells. Cell Oncol (Dordr). 2017;40(5): $457-470$ 
10. Zhang M, Gao CE, Chen WL, et al. Opposite response to hypoxia by breast cancer cells between cell proliferation and cell migration: a clue from microRNA expression profile. Oncol Lett. 2018;15(3): 2771-2780.

11. Kapinova A, Kubatka P, Zubor P, et al. The hypoxia-responsive long non-coding RNAs may impact on the tumor biology and subsequent management of breast cancer. Biomed Pharmacother. 2018;99:51-58.

12. Tang ZN, Zhang F, Tang P, Qi XW, Jiang J. Hypoxia induces RANK and RANKL expression by activating HIF-1 $\alpha$ in breast cancer cells. Biochem Biophys Res Commun. 2011;408(3):411-416.

13. Zhou Z, Wang S, Song C, Hu Z. Paeoniflorin prevents hypoxia-induced epithelial-mesenchymal transition in human breast cancer cells. Onco Targets Ther. 2016;9:2511-2518.

14. Jeong YJ, Cho HJ, Magae J, Lee IK, Park KG, Chang YC. Ascofuranone suppresses EGF-induced HIF-1 $\alpha$ protein synthesis by inhibition of the Akt/mTOR/p70S6K pathway in MDA-MB-231 breast cancer cells. Toxicol Appl Pharmacol. 2013;273(3):542-550.

15. Li J, Zhang C, Jiang H, Cheng J. Andrographolide inhibits hypoxiainducible factor-1 through phosphatidylinositol 3-kinase/AKT pathway and suppresses breast cancer growth. Onco Targets Ther. 2015;8: 427-435.

16. Ghattass K, El-Sitt S, Zibara K, et al. The quinoxaline di-N-oxide DCQ blocks breast cancer metastasis in vitro and in vivo by targeting the hypoxia inducible factor-1 pathway. Mol Cancer. 2014;13:12.

17. Tang XD, Zhou X, Zhou KY. Dauricine inhibits insulin-like growth factor-I-induced hypoxia inducible factor 1 alpha protein accumulation and vascular endothelial growth factor expression in human breast cancer cells. Acta Pharmacol Sin. 2009;30(5):605-616.
18. Sim MW, Cohen MS. The discovery and development of vandetanib for the treatment of thyroid cancer. Expert Opin Drug Discov. 2014; 9(1):105-114.

19. Grabowski P, Briest F, Baum RP, et al. Vandetanib therapy in medullary thyroid cancer. Drugs Today (Barc). 2012;48(11):723-733.

20. Thornton K, Kim G, Maher VE, et al. Vandetanib for the treatment of symptomatic or progressive medullary thyroid cancer in patients with unresectable locally advanced or metastatic disease: U.S. Food and Drug Administration drug approval summary. Clin Cancer Res. 2012;18(14): 3722-3730

21. Zhou Y, Zhang Y, Zou H, et al. The multi-targeted tyrosine kinase inhibitor vandetanib plays a bifunctional role in non-small cell lung cancer cells. Sci Rep. 2015;5:8629.

22. Li G, Shan C, Liu L, et al. Tanshinone IIA inhibits HIF-1 $\alpha$ and VEGF expression in breast cancer cells via mTOR/p70S6K/RPS6/4E-BP1 signaling pathway. PLoS One. 2015;10(2):e0117440.

23. Lodola F, Laforenza U, Cattaneo F, et al. VEGF-induced intracellular $\mathrm{Ca}^{2+}$ oscillations are down-regulated and do not stimulate angiogenesis in breast cancer-derived endothelial colony forming cells. Oncotarget. 2017;8(56):95223-95246.

24. Sohn EJ, Jung DB, Lee H, et al. CNOT2 promotes proliferation and angiogenesis via VEGF signaling in MDA-MB-231 breast cancer cells. Cancer Lett. 2018;412:88-98.

25. Clemons MJ, Cochrane B, Pond GR, et al. Randomised, phase II, placebo-controlled, trial of fulvestrant plus vandetanib in postmenopausal women with bone only or bone predominant, hormone-receptorpositive metastatic breast cancer (MBC): the OCOG ZAMBONEY study. Breast Cancer Res Treat. 2014;146(1):153-162.
OncoTargets and Therapy

\section{Publish your work in this journal}

OncoTargets and Therapy is an international, peer-reviewed, open access journal focusing on the pathological basis of all cancers, potential targets for therapy and treatment protocols employed to improve the management of cancer patients. The journal also focuses on the impact of management programs and new therapeutic agents and protocols on

\section{Dovepress}

patient perspectives such as quality of life, adherence and satisfaction. The manuscript management system is completely online and includes a very quick and fair peer-review system, which is all easy to use. Visit http://www.dovepress.com/testimonials.php to read real quotes from published authors. 